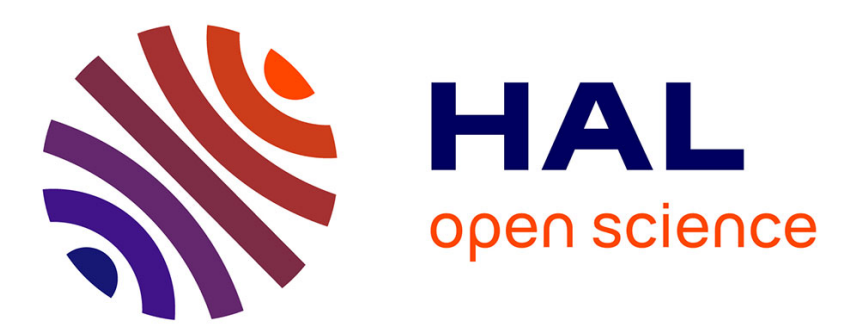

\title{
Delay stabilizes stochastic systems near an non-oscillatory instability
}

Axel Hutt, Jérémie Lefebvre, André Longtin

\section{To cite this version:}

Axel Hutt, Jérémie Lefebvre, André Longtin. Delay stabilizes stochastic systems near an nonoscillatory instability. EPL - Europhysics Letters, 2012, 98 (2), pp.200004. 10.1209/02955075/98/20004 . hal-00710426

\section{HAL Id: hal-00710426 \\ https://hal.inria.fr/hal-00710426}

Submitted on 20 Jun 2012

HAL is a multi-disciplinary open access archive for the deposit and dissemination of scientific research documents, whether they are published or not. The documents may come from teaching and research institutions in France or abroad, or from public or private research centers.
L'archive ouverte pluridisciplinaire HAL, est destinée au dépôt et à la diffusion de documents scientifiques de niveau recherche, publiés ou non, émanant des établissements d'enseignement et de recherche français ou étrangers, des laboratoires publics ou privés. 


\title{
Delay stabilizes stochastic systems near an non-oscillatory insta- bility
}

\author{
Axel HutT ${ }^{1}$, JÉrémie Lefebvre ${ }^{2}$ and André LONGTiN ${ }^{3}$ \\ 1 INRIA CR Nancy - Grand Est, Team CORTEX, 54600 Villers-les-Nancy, France \\ 2 Sprott Center, 501 Smyth Road, Ottawa, Canada \\ 3 Department of Physics, University of Ottawa, 50 Louis Pasteur, Ottawa, Ontario, K1N-6N5, Canada
}

PACS 02.50.Fz - Stochastic analysis

PACS 02.30.Ks - Delay and functional equations

PACS 87.19.1c - Noise in the nervous system

\begin{abstract}
The work discovers a stochastic bifurcation in delayed systems in the presence of both delay and additive noise. To understand this phenomenon we present a stochastic center manifold method to compute a non-delayed stochastic order parameter equation for a scalar delayed system driven by additive uncorrelated noise. The derived order parameter equation includes additive and multiplicative white and coloured noise. An illustrative neural system with delayed self-excitation reveals stationary states that are postponed by combined additive noise and delay. A final brief analytical treatment of the derived order parameter equation reveals analytically the shift of the stationary states which depends on the delay and the noise strength.
\end{abstract}

Nonlinear time-delayed systems arise in numerous areas [1]. The effect of noise on such systems is of great interest, for example in the context of delayed laser dynamics [2]. Biological systems are rife with delays, and noise is often significant enough to e.g. alter bifurcations in genetic networks [3], even producing stationary states - as maxima of the stationary probability density - that have no deterministic counterpart [4]. The same is true for neural systems involved in the control of e.g. the pupil [5], eye movements [6], balance [7] and neural rhythms [8]. Analytical insights into the properties of stochastic delaydifferential systems (SDDEs) have been hindered by the fact that Fokker-Planck analysis is not valid for such nonMarkovian dynamics. Advances have relied on approximations using e.g. small delay expansions [9], multiscale methods [10], delayed random walks [11], as well as coarsegrained dynamics [12] naturally applied to intrinsically bistable delayed systems.

Yet a general theory for SDDEs is still lacking, especially near instabilities. A technique to calculate the effects of noise well into nonlinear regimes would be an important step, and is the focus of our Letter. We highlight delayed dynamics involving a pitchfork bifurcation, but our work is in fact applicable to all systems having a one-dimensional normal form in the absence of delay. We consider the system

$$
\frac{d x(t)}{d t}=-x(t)+\beta x(t-\tau)-\gamma x^{3}(t-\tau)+\kappa \xi(t)
$$

with $\beta, \gamma \in \mathcal{R}$ and the delay $\tau$. Here $\xi(t)$ is Gaussian white noise $(\mathrm{GWN})$ with $\langle\xi(t)\rangle=0,\left\langle\xi(t) \xi\left(t^{\prime}\right)\right\rangle=2 \delta\left(t-t^{\prime}\right)$. The noise strength is denoted by $\kappa$, and $\langle\cdot\rangle$ denotes the ensemble average. A motivation for our work arises in the context of a single neuron with global excitatory delayed feedback from a population, which exhibits a spatially synchronized mean membrane potential $U(t)$ obeying

$$
\frac{d U(t)}{d t}=-\alpha U(t)+K_{0} S\left[U\left(t-\tau_{0}\right)\right]+E_{0}+\xi(t)
$$

The parameter $\alpha$ is the mean decay rate of synaptic responses and $K_{0}$ represents the excitatory feedback level. The sigmoid function $S(V)=S_{0} /\left(1+\exp \left(-c\left(V-V_{t h r}\right)\right)\right)$ reflects the population firing rate function with constant parameters $S_{0}, V_{t h r}$ and $c$ and $\tau_{0}$ denotes the fixed feedback delay. The global external stimulus includes the mean background activity $E_{0}$ from external neural processes, and the small input $\xi(t)$ represents random fluctuations around that mean [8]. Equation (1) includes the pre-factors $\beta=S^{0} K_{0} c / 4 \alpha>0, \gamma=S^{0} K_{0} c^{3} / 48 \alpha>0$ and the rescaled time and delay $t \rightarrow t / \alpha$ and $\tau=\alpha \tau_{0}$, respectively. The pre-factors $\beta$ and $\gamma$ are related by $\gamma=\eta \beta^{3}$ 
with $\eta=4\left(\alpha / S_{0} K_{0}\right)^{2} / 3$. This dependence of linear and nonlinear pre-factors in Eq. (1) is a generic property of neural systems. Considering small deviations of $U(t)$ from $V_{t h r}$, i.e. $x(t) \equiv U(t)-V_{t h r},|x(t)| \ll V_{t h r}$, and Taylorexpanding $S$ about $V_{t h r}$ up to cubic order, yields the delayed Langevin equation Eq. (1). The resting states of Eq. (1) for zero noise are $x_{0}=0, x_{1,2}= \pm \sqrt{\varepsilon / \eta(\varepsilon+1)^{3}}$ with the new control parameter $\varepsilon=\beta-1>0$. We find the well-expected pitchfork bifurcation for small $\varepsilon$. Moreover $x_{0}$ is metastable for $\varepsilon=0$.

Figure 1 shows the stationary probability density $P_{s}(x)$ of the stochastic process (1) for vanishing delay (Fig. 1(a)) and a large delay $\tau=2$ (Fig. 1(b)). For $\tau=0$, we observe the expected uni-modal density for $\varepsilon<0$ and the bi-modal density for $\varepsilon>0$. In the case of a large delay $\tau=2$ the bi-modal density emerges for much larger values of $\varepsilon$ compared to the case $\tau=0$. This indicates that the critical instability point is moved to larger values of the control parameter, i.e. the system exhibits stationary activity about the state $x=0$ for larger control parameters than expected from a deterministic analysis. Moreover, it is well-known that noise-free delayed scalar systems such as (1) for $\kappa=0$ [13] and stochastic non-delayed scalar systems with additive GWN, i.e. (1) with $\tau=0$, have identical stationary states, cf. dotted line in Fig. 1. Consequently the stochastic bifurcation shown in Fig. 1(b) occurs only in the presence of both delay and additive noise. Hence introducing delay in a system subjected to additive noise stabilizes the system. Such a noise-induced transition is known only for multiplicative GWN noise in delayed systems $[5,14]$. Numerical work had also shown that additive GWN can shift a Hopf bifurcation in a scalar SDDE [5], and here we provide analytics that explain a novel and simpler manifestation of this phenomena.
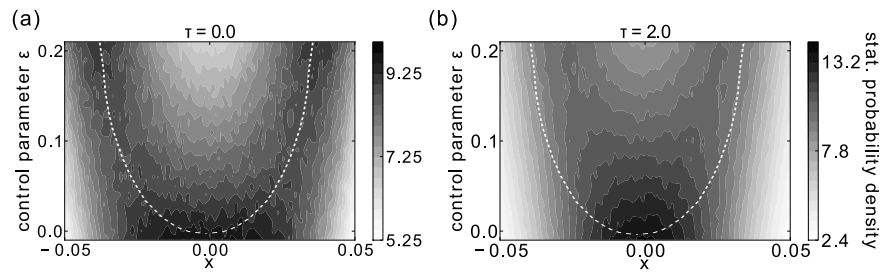

Fig. 1: The stationary probability density (PDF) of the stochastic process (1) computed numerically without (a) and with delay (b). The white dotted line denotes the stationary states of (1) in the absence of both delay and noise. Simulations used a delayed Euler-Maruyama integration method with iteration step $d t=0.01$ and parameters $\eta=80$ (used throughout) and $\kappa=0.015$. The PDFs were computed using an ensemble average of $15 \cdot 10^{4}$ trajectories in the stationary state reached at $t=1000$.

To investigate this numerical finding analytically, the constant control parameter $\varepsilon$ is made a dynamic variable with $d \varepsilon / d t=0$, allowing an easier application of the center manifold approach. This yields the augmented system

$$
\frac{d x(t)}{d t}=-x(t)+x(t-\tau)+F(x, \varepsilon, t), \frac{d \varepsilon}{d t}=0
$$

with $F(x, \varepsilon, t)=\varepsilon x(t-\tau)-\eta(1+\varepsilon)^{3} x^{3}(t-\tau)+\kappa \xi(t)$ including both the nonlinear interaction terms and the random input. Moreover, the linear terms no longer depend on $\varepsilon$. Mathematically, the system in Eq. (3) has two variables but evolves in an infinite-dimensional space due to the delay. Hence it is practical to introduce the 2-dimensional vector function $z_{t}(\theta)=(x(t+\theta), \varepsilon)^{T}=\left(z_{t}(\theta)_{1}, z_{t}(\theta)_{2}\right)^{T}$ with $-\tau \leq \theta \leq 0$, where $z_{t}$ lives in the Banach space of continuous maps $\mathcal{C}_{B} \equiv C([-\tau, 0], \mathbb{R} \times \mathbb{R})[15,16]$. Then (3) reads

$$
\frac{d}{d t} z_{t}(\theta)=\mathcal{A}\left(z_{t}(\theta)\right)+X_{o} F\left[z_{t}, t\right]
$$

where $\mathcal{A}=\partial z_{t} / \partial \theta$ for $-\tau \leq \theta<0, \mathcal{A}=L\left[z_{t}\right]$ for $\theta=0$, with the linear operator $L\left[z_{t}\right]=\left(-z_{t}(0)_{1}+z_{t}(-\tau)_{1}, 0\right)^{T}$ which is re-formulated as functional operator

$$
L\left[z_{t}\right]=\int_{-\tau}^{0}\left(\begin{array}{cc}
-\delta(\theta)+\delta(\theta+\tau) & 0 \\
0 & 0
\end{array}\right) z_{t}(\theta) d \theta .
$$

The function $F$ is re-written in the new variable $z_{t}$ as $F(x, \varepsilon, t)=\left(z_{t}(0)_{1} z_{t}(0)_{2}-\eta\left(1+z_{t}(0)_{2}\right)^{3} z_{t}^{3}(-\tau)_{1}+\right.$ $I(t), 0)^{T}$, i.e. it is a non-linear functional of $z_{t}[15,16]$

$$
\begin{gathered}
F\left[z_{t}, t\right]=\int_{-\tau}^{0} \int_{-\tau}^{0} \omega_{2}\left(\theta, \theta^{\prime}\right) z_{t}(\theta) \otimes z_{t}\left(\theta^{\prime}\right) d \theta d \theta^{\prime} \\
+\int_{-\tau}^{0} \int_{-\tau}^{0} \int_{-\tau}^{0} \omega_{3}\left(\theta, \theta^{\prime}, \theta^{\prime \prime}\right) z_{t}(\theta) \otimes z_{t}\left(\theta^{\prime}\right) \otimes z_{t}\left(\theta^{\prime \prime}\right) d \theta d \theta^{\prime} d \theta^{\prime \prime} \\
+(I(t), 0)^{t}
\end{gathered}
$$

with corresponding tensors $\omega_{2} \in \mathbb{R}^{2 \times 2}, \omega_{3} \in \mathbb{R}^{2 \times 2 \times 2}$ and the dyadic product $\otimes$. Moreover to gain a compact description the connection function $X_{o}(\theta)$ was introduced $a d$ hoc in Eq. (4) as $X_{o}(\theta)=0$ for $-\tau \leq \theta<0, X_{o}(0)=\mathbb{I}_{2}$ with the $2 \times 2$ identity matrix $\mathbb{I}_{2}[15,16]$. Consequently, considering the functionals $L\left[z_{t}\right]$ and $F\left[z_{t}, t\right]$ and the connection function $X_{o}(\theta)$ the original scalar problem is reformulated in the infinite-dimensional function $z_{t}$ and the system obeys Eq. (4).

The subsequent mathematical treatment is structured as follows. We first solve the linear eigenvalue problem in (4), i.e. $F=0$, and project the nonlinear dynamics onto the corresponding (linear) eigenvectors. The augmented system is constructed in such a way that a subset of the eigenspectrum exhibits a vanishing real part and the complementary subset has non-vanishing real parts. Hence it is possible to split the dynamics into two parts, namely the center and the stable part. To reduce the dimension of the still infinite-dimensional system, we introduce a nonautonomous center manifold approach and treat the random input similarly to the nonlinear part.

In more detail, the linear eigenspectrum of $\mathcal{A}$ is defined by the characteristic equation $\lambda=-1+\exp (-\lambda \tau)$ with the eigenvalues $\lambda \in \mathcal{C}$. We find $\lambda_{0}=0$ plus infinitely many complex conjugate roots $\lambda_{n}, n>0$ with $\operatorname{Re}\left(\lambda_{n}\right)<0$. 
The corresponding eigenbasis of $\mathcal{A}$ is called $\Phi(\theta)$. Consequently, the phase space splits into a center space $\mathcal{U}$ spanned by vectors with the eigenbasis $\Phi_{u}(\theta)$ corresponding to $\lambda_{0}$ and the stable space $\mathcal{S}$ with the corresponding eigenbasis $\Phi_{s}(\theta)$.

The adjunct eigenvalue problem for $t \rightarrow-t$ yields the adjunct eigenbasis $\Psi(\theta)$ that is bi-orthonormal to $\Phi$. To define the metric in the Banach space, a specific bilinear form $(\cdot, \cdot)[15,16]$ defines the equivalent of a scalar product in a vector space. We find $\left(\Psi_{u}, \Phi_{u}\right)=\mathbb{I}_{2}$ with the eigenbasis $\Phi_{u}(\theta) \in \mathcal{U}$ of the adjunct problem. Accordingly, the center and stable eigenbases are bio-orthogonal with $\left(\Psi_{u}, \Phi_{s}\right)=0$. Projections onto the center space are given by the projector operator $P(\cdot) \equiv \Phi_{u}\left(\Psi_{u}, \cdot\right)[15,16]$ which reads for $(1)$

$$
P(\phi(\Theta))=(1+\tau)^{-1}\left(\phi(0)+\int_{-\tau}^{0} \phi(s) d s\right) .
$$

Then one can decompose $z_{t} \in \mathcal{U} \times \mathcal{S}$ into the center contribution $(u(t), \varepsilon)^{T}=P\left(z_{t}\right) \in \mathcal{U}$ with the order parameter $u \in \mathcal{R}$ and a stable contribution $s_{t}(\theta)=(\mathbb{I}-P)\left(z_{t}\right) \in \mathcal{S}$, i.e. $z_{t}(\theta)=(u(t), \varepsilon)^{T}+s_{t}(\theta)$. Consequently the corresponding projected dynamics in $\mathcal{U}$ and the complementary space $\mathcal{S}$ are given by

$$
\begin{aligned}
\frac{d u(t)}{d t} & =(1+\tau)^{-1} F\left[u+s_{t}, \varepsilon, t\right] \quad, \quad \frac{d \varepsilon(t)}{d t}=0(6) \\
\frac{d}{d t} s_{t}(\theta) & =\mathcal{A}\left(s_{t}\right)+\left(X_{o}-\frac{1}{1+\tau}\right) F\left[u+s_{t}, \varepsilon, t\right] .(7)
\end{aligned}
$$

This formulation (6), (7) of the original problem (1) is still infinite dimensional, but allows for a reduction in dimension by center manifold theory, where the dynamics of the stable projection modes are expressed as functions of the center projection modes, i.e. $s_{t}(\theta, t)=s_{t}(u, \varepsilon, \theta, t)$. In autonomous delayed systems the existence of this relation has been proven mathematically [17]. Motivated by the existence of stochastic center manifolds in non-delayed systems [19], the present work proposes the following approximation scheme. The small random input is assumed to add a small perturbation to the center manifold obtained for the deterministic (i.e. $\kappa=0$ ) delayed system, which is independent of the center manifold. Specifically, we assume that the stochastic variation of the center manifold just depends on the delays but is independent of the order parameter $u$ and the control parameter, i.e.

$$
s_{t}(\theta)=h_{\text {det }}(u, \varepsilon, \theta)+h_{t}(\theta, t)
$$

with the deterministic center manifold contribution $h_{\text {det }}$ and the stochastic perturbation $h_{t}$. In addition, we assume that the stable (nonlinear) projection modes $s_{t}$ fully evolve in the stable (linear) subspace, i.e. $P\left(s_{t}\right)=0$. For later computations, it is useful to implement this assumption by $P\left(h_{\text {det }}\right)=0$ and $h_{t}=(\mathbb{I}-P) H_{t}$ with an auxilliary function $H_{t}=H(t+\theta) \in \mathcal{C}_{B}$. This auxilliary function will be defined later.
We first compute the deterministic center manifold $h_{\text {det }}$ according to standard techniques $[15,16]$, i.e. $s_{t}=h_{\text {det }}$. It is important to note that, by virtue of the extension of (1) to the augmented system in Eq. (3), the relation $s_{t}=h_{\text {det }}$ is exact [17] for all values of the control parameter $\varepsilon$ in the absence of noise, i.e. $\kappa=0$. Inserting Eqs. (6), (7) into (8) yields the partial differential equation for $h_{\text {det }}$ in $u$ and $\theta$

$$
\begin{aligned}
& \frac{1}{1+\tau} \frac{\partial h_{d e t}}{\partial u} F\left[u+h_{d e t}\right]= \\
& \mathcal{A}\left(h_{d e t}\right)+\left(X_{o}-\frac{1}{1+\tau}\right) F\left[u+h_{d e t}\right] .
\end{aligned}
$$

To solve this equation, typically one considers the activity being close to the resting state $u=0$ and chooses the bi-polynomial ansatz $h_{\text {det }}=h_{2}\left(u^{2}, u \varepsilon, \varepsilon^{2}, \theta\right)+$ $h_{3}\left(u^{3}, u^{2} \varepsilon, u \varepsilon^{2}, \varepsilon^{3}, \theta\right)+\cdots$. Assuming that the order parameter has the same order of magnitude as the control parameter, we introduce the scaling $u \sim \mathcal{O}(\varepsilon)$ which defines the order of the terms to $h_{n} \sim \mathcal{O}\left(\varepsilon^{n}\right)$. Consequently, for small order parameters $u$ it is sufficient to compute just a few lower order terms. Applying the aforementioned necessary condition $P\left(h_{n}\right)=0$ up to order $\mathcal{O}\left(\varepsilon^{4}\right)$, the deterministic center manifold reads

$$
h_{\text {det }} \approx\left(a \varepsilon+b_{3} \varepsilon^{2}+b_{4} \varepsilon^{3}\right) u+\left(d_{3}+d_{4} \varepsilon\right) u^{3}
$$

with functions $a=a(\theta, \tau), b_{i}=b_{i}(\theta, \tau), d_{i}=d_{i}(\theta, \tau)$. In addition $a, b_{i}, d_{i} \rightarrow 0$ for $\tau \rightarrow 0$, i.e. the center manifold vanishes for vanishing delay. This reflects the fact that there is no time-scale separation in the dynamics of the non-delayed system.

Now let us consider the non-autonmous center manifold perturbation $h_{t}$. Assuming that the stochastic perturbations are small compared to $u$ and $\varepsilon$, i.e. $h_{t}, \kappa \sim \mathcal{O}\left(\varepsilon^{2}\right)$, and inserting Eqs. (6), (7) into (8) utilizing $d s_{t} / d t=$ $\left(\partial h_{\text {det }} / \partial u\right)(d u / d t)+\partial h_{t} / \partial t$, the dynamics of the stochastic center manifold contribution $h_{t}$ separates from the autonomous dynamics at lowest order $\mathcal{O}\left(\varepsilon^{2}\right)$ and obeys

$$
\frac{\partial h_{t}}{\partial t}=\mathcal{A}\left(h_{t}\right)+\kappa\left(X_{o}-\frac{1}{1+\tau}\right) \xi(t) .
$$

Then recalling the ansatz $h_{t}=(\mathbb{I}-P) H(t+\theta)$ yields the linear delay equation

$$
d H(t+\theta) / d t=L[H]+\kappa \xi(t) .
$$

with $L[H]=-H(t)+H(t-\tau)$. It is interesting to mention that (10) is valid for all delayed systems exhibiting a one-dimensional normal form (proof not shown). Hence, the stochastic perturbation of the center manifold $h_{t}$ is independent of the nonlinear interactions in the system. The solution of Eq. (10) reads

$$
\begin{aligned}
H(t) & =\kappa \int_{0}^{t} H_{0}(t-s) d W(s), t \geq 0 \\
H_{0}(t) & =\frac{1}{2 \pi(1+\tau)}+\sum_{n=1}^{\infty} \frac{\exp \left(\lambda_{n} t\right)}{1+\tau \exp \left(-\lambda_{n} \tau\right)}+c .
\end{aligned}
$$


for initial conditions $H(\theta)=0,-\tau \leq \theta \leq 0$. Here $W(t)$ is the Wiener process corresponding to the random input $\xi(t)$ and $H_{0}(t)$ is the characteristic function of (10) and $\lambda_{n}, \operatorname{Re}\left(\lambda_{n}\right)<0$ are the characteristic roots of the linear problem. Essentially, inserting $H(t)$ into $h_{t}=(\mathbb{I}-$ $P) H(t+\tau)$ utilizing the definition of the projector $P$ in Eq. (5) gives explicitely the non-autonomous perturbation of the center manifold

$$
\begin{aligned}
& h_{t}(\theta, t)=\kappa \int_{0}^{t+\theta} H_{0}(t+\theta-s) d W(s) \\
& -\frac{\kappa}{1+\tau} \int_{0}^{t} H_{0}(t-s) d W(s) \\
& -\frac{\kappa}{1+\tau} \int_{-\tau}^{0} \int_{0}^{t+s} H_{0}(t+s-r) d W(r) d s .
\end{aligned}
$$

With $s_{t}=h_{\text {det }}+h_{t}$ Eq. (6) becomes the final order parameter equation

$$
\begin{aligned}
& \frac{d u(t)}{d t}=\frac{\kappa}{1+\tau} \xi(t)+A_{0} Z(t)+B_{0} Z^{3}(t) \\
& +\left(A_{1}+B_{1} Z(t)+C Z^{2}(t)\right) u+\left(A_{3}+B_{3} Z^{2}(t)\right) u^{3} \\
& +A_{5} u^{5}+A_{7} u^{7}+A_{9} u^{9},
\end{aligned}
$$

where $Z(t)=h_{t}(t,-\tau)$ and $A_{n}(\varepsilon, \tau), B_{n}(\varepsilon, \tau), C(\varepsilon, \tau)$ are constants. We observe that vanishing noise $\kappa=0$ sets $Z(t)=0$ and the stable dynamics obeys the deterministic center manifold with $h=h_{\text {det }}$. Additional numerical simulations of the original and the reconstructed solution in the absence of noise have shown a perfect agreement of $x(t)$ and $u(t)$ (result not shown). This finding, that the deterministic center manifold does not affect the dynamics of the original system, is in accordance with a previous study [16].

According to (11) and (12), the center manifold perturbation $Z$ evolves on infinite many time scales and represents a coloured-noise process. Equation (13) reveals that the nonlinear delayed system responds to the additive random process $\xi(t)$ by generating coloured noise $Z(t)$ that modifies the original additive input and renders the system parameters stochastic, i.e. introduces multiplicative noise. This multiplicative noise is well-known to produce noiseinduced transitions and we understand immediately the shift observed in Fig. 1. For clarification, the multiplicative noise originates from the stochasticity of the stable modes $s_{t}$ which couples into the order parameter dynamics. We point out that this novel mechanism is generic in the sense that it occurs in all delayed systems subjected to additive noise which are close to an non-oscillatory instability. Moreover, the specific effect of additive noise on the system depends strongly on the nonlinear order of the system. For instance, in delayed systems involving a quadratic nonlinearity on the center manifold the additive noise induces an additional constant [20] and the center manifold in the absence of noise affects the deterministic dynamics as shown in a previous study [18].
To evaluate the proposed reduction scheme, recall the split of $z_{t}(\theta)$ into a center manifold and a stable manifold contribution. Applying the center manifold reduction scheme, for $\theta=0$ this split reads $z_{t}(\theta=0)=$ $u(t)+h_{\text {det }}(u, \varepsilon, 0)+h_{t}(t, 0)=x(t)$ where the last equation results from the general definition of $z_{t}$. This relation gives the exact rule of reconstruction of the original process $x(t)$ by $u(t)$ and the corresponding center manifold. Now a reduced description $x(t) \approx u(t)$, where $u$ obeys Eq. (13), is valid if $h(t, 0)=h_{\text {det }}(u, \varepsilon, 0)+h_{t}(t, 0) \ll u(t)$, i.e. the nondelayed order parameter equation (13) approximates well the original systems dynamics of Eq. (1). The variance of the stable manifold $\left\langle h^{2}(t, 0)\right\rangle$, i.e. the reconstruction error made by the reduction of $x$ to $u$, reads

$\left\langle h^{2}(t, 0)\right\rangle=\left\langle h_{\text {det }}^{2}(0)\right\rangle+a \kappa^{2} \tau^{3} /(1+\tau)^{4}+\kappa^{2} R\left(\tau, 1 / \lambda_{n}\right)$

with a pre-factor $a$ and the remaining term $R\left(\tau, 1 / \lambda_{n}\right) \rightarrow 0$ for $\tau \rightarrow 0$. Here $\langle\cdot\rangle$ denotes the ensemble average. Since $\left\langle h_{\text {det }}^{2}(0)\right\rangle \rightarrow 0$ for $\tau \rightarrow 0$, the reconstruction of $x$ is perfect for vanishing delay or noise. For small but non-vanishing delay or noise, the original delayed dynamics is still wellapproximated by the order parameter equation (13).

Similar to previous studies on the deterministic Hopf

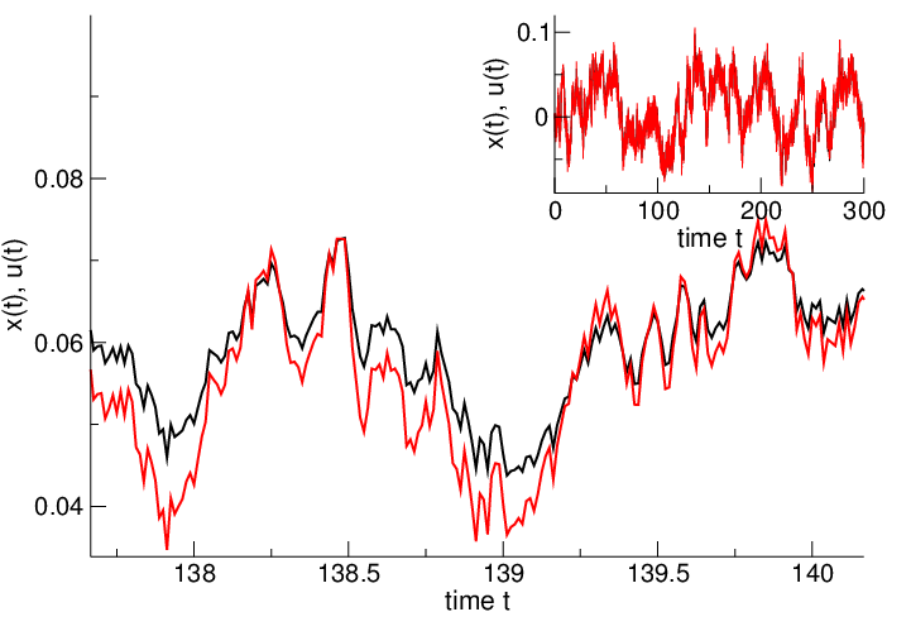

Fig. 2: Comparison of original and reconstructed stochastic paths. The original trajecory $x(t)$ (red) has been computed by numerical integration of Eq. (1) and the reconstructed trajectory $u(t)$ (black) obeys Eq. (13). The inset shows the fulllength trajectories while the large panel presents a focus on a small time interval. Parameters are $\epsilon=0.2, \tau=0.5, \kappa=0.02$ and $d t=0.0125$, other parameters are taken from Fig. 1.

bifurcation [16,18], Fig. 2 compares the original and reconstructed solution of Eq. (1) and (13) by plotting a simulated single path of $x(t)$ and $u(t)$. It reveals a very good fit of $x(t)$ and $u(t)$ which affirms the validity of the proposed reduction scheme.

To gain even deeper insight into the reduced dynamics (13), Fig. 3 compares the numerically estimated stationary probability density functions (PDF) of the original delayed process (1) and the non-delayed order parameter process (13) for various noise strengths $\kappa$ and delays 
(a)
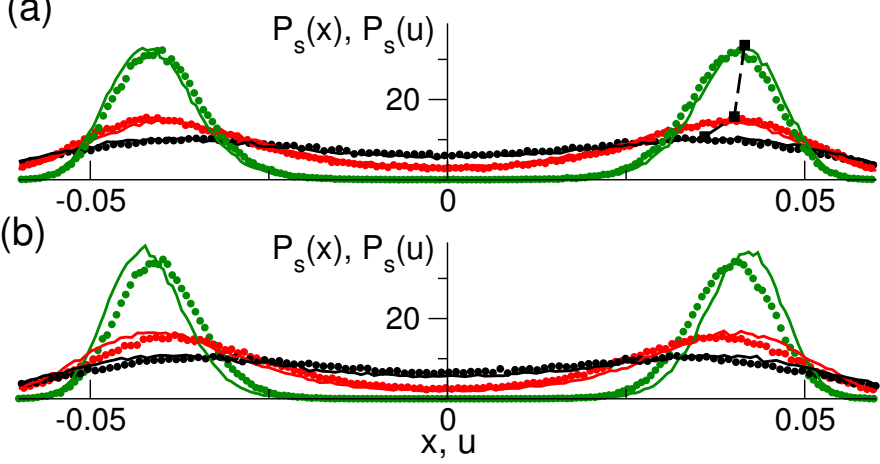

Fig. 3: The order parameter $u$ reconstructs well the original amplitude $x$ in the stationary state near the bifurcation $(\varepsilon=$ $0.3)$. The PDFs $P_{s}(u)$ and $P_{s}(x)$ are computed from numerical solutions of Eq. (13) (solid) and (1) (dotted), respectively, for (a) $\tau=0.5$, (b) $\tau=1.0$. Noise strengths are $\kappa=0.005$ (green), $\kappa=0.01$ (red) and $\kappa=0.015$ (black). In (a) the PDF maxima are linked (dashed line) to show the shift to lower amplitudes as $\kappa$ increases. The numerical scheme is identical to the scheme used in Fig. 1.

$\tau$. The corresponding PDFs show very good accordance for $\tau=0.5$ (Fig. 3(a)), whereas the larger delay $\tau=1.0$ shown in Fig. 3(b) yields a larger but still reasonable reconstruction error. This result confirms quantitatively the validity of the employed center manifold reduction scheme for small delays.

Moreover, we observe that the magnitude of the maxima of the PDFs decrease while increasing the noise strength $\kappa$, cf. dashed line in Fig. 3(a). Figure 4 explores this shift in more detail by plotting the location of maxima of the PDFs for various control parameters and reveals a noiseinduced shift to larger values of $\varepsilon$ (solid lines and symbols). This finding affirms further the stabilization effect of the additive random process shown in Fig. 1 in the presence of delays.

To quantify the shift of the maxima analytically, we introduce a new random replacement process $Z_{0}(t) \approx Z(t)$ that shares the first two statistical moments with $Z(t)$ for $t \rightarrow$ $\infty$, i.e. $\left\langle Z_{0}(t)\right\rangle=0,\left\langle Z_{0}^{2}(t)\right\rangle=\sigma^{2}$ with the variance $\sigma^{2}=$ $\left\langle h_{t}^{2}(t,-\tau)\right\rangle$. This variance is small for small delays and noise strengths (proof not shown). The advantage of introducing this approximative process is the fact that $Z(t)$ is rather complex involving many time scales and $Z_{0}$ allows for simpler analytical treatment compared to the original process. We assume that $Z_{0}(t)$ obeys the OrnsteinUhlenbeck process $d Z_{0}=-\left(Z_{0} / \sigma^{2}\right) d t+d W_{0}(t)$ with the Wiener process $W_{0}(t),\left\langle d W_{0}(t)\right\rangle=0,\left\langle d W_{0}(t) d W_{0}\left(t^{\prime}\right)\right\rangle=$ $2 \delta\left(t-t^{\prime}\right) d t d t^{\prime}$. By virtue of the small values of $\sigma^{2}$, we explore the time scale separation between $u$ and $Z_{0}$ and apply an adiabatic elimination scheme [21] to Eq. (13). This allows to derive the Fokker-Planck equation for the

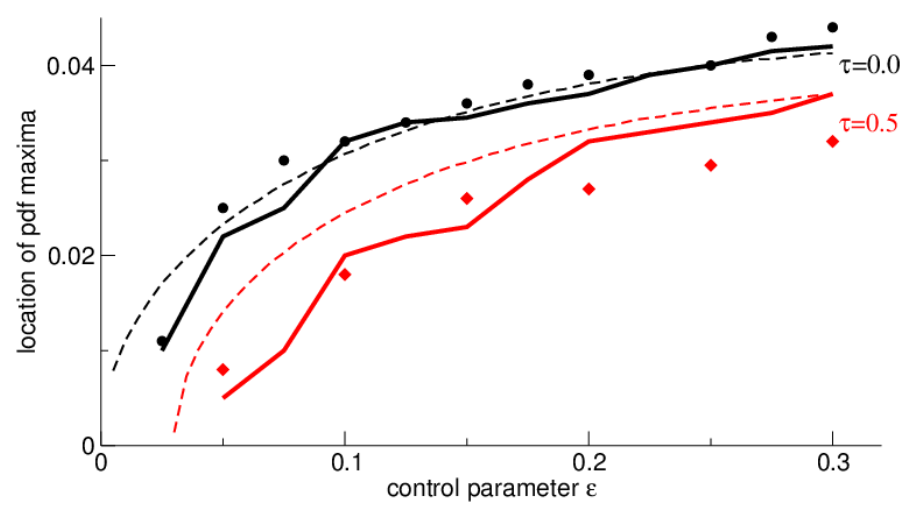

Fig. 4: The location of the maxima of the stationary probability $P_{s}(u)$ is shifted to larger values in the presence of delay. The locations are estimated numerically from the probility density gained by numerical simulations of the original delay equation (1) (circles and diamonds) and the derived non-delayed equation (13) (solid lines). For comparison, the dashed lines denote the analytical maxima $x_{n}$ gained from Eq. (14).

probability density $P(u, t)$ of the order parameter $u(t)$ to

$$
\begin{aligned}
& \frac{\partial P(u, t)}{\partial t}=-\frac{\partial}{\partial u}\left(\left(A_{1}+A_{1, s h i f t}\right) u+\left(A_{3}+A_{3, s h i f t}\right) u^{3}\right. \\
& \left.+A_{5} u^{5}+A_{7} u^{7}+A_{9} u^{9}\right) P(u, t)+D \frac{\partial^{2}}{\partial u^{2}} P(u, t)
\end{aligned}
$$

with $A_{1, \text { shift }}=C \sigma^{2}, A_{3, \text { shift }}=B_{3} \sigma^{2}, C$ and $B_{3}$ taken from Eq. (13) and $D=\kappa^{2} /(1+\tau)^{2}$. We observe a noise-dependent additive constant in the linear and cubic terms, and hence a shift of the stationary state of the system by $A_{1, \text { shift }}$. In addition $C=C(\varepsilon, \tau) \rightarrow 0$ for $\tau \rightarrow 0$, i.e. the noise-induced effect vanishes for $\tau=0$. Since $C<0$, it is $A_{1, \text { shift }}<0$ and the effective stability threshold is shifted to larger values of $u$. It is easy to find analytically the stationary solution $P_{s}(u)$ of (14) and Fig. 4(dashed lines) shows the locations $x_{n}$ of the local maxima of $P_{s}(u)$. As in the numerical results, the delay induces a shift of the probability density to larger magnitudes. Figure 4 also reveals that the delay-induced shift of the original system (symbols) is larger than the shifts found analytically (lines) for larger $\varepsilon$, i.e. the analytical results underestimate the delay-induced shift.

In summary, the work discovers a delay-induced shift of stability in delayed systems driven by additive uncorrelated noise. To explain this phenomenon, we introduce an analytical approach to derive a stochastic non-delayed order parameter equation for a scalar SDDE. This new equation involves both white and coloured noises. For the delayed pitchfork system, a comparison of probability densities reveal close numerical agreement of the nondelayed order parameter equation and the original system. A brief analytical discussion of the probability density of the non-delayed order parameter dynamics affirms the results. Importantly both delay and additive noise affects the presence of a stationary solution at the origin close to 
an instability even though this instability is not caused by the delay.

JL and AL would like to thank NSERC and FQRNT for support. AH acknowledges funding from the the European Research Council for support under the European Union's Seventh Framework Programme (FP7/2007-2013)/ ERC grant agreement no. 257253.

\section{REFERENCES}

[1] T. Erneux, Applied Delay-Differential Equations (Springer, New York, 2009)

[2] J.M. Buldu, J. Garcia-Ojalvo and M.C. Torrent, Phys. Rev. E 69, 046207 (2004)

[3] D. Bratsun et al., PNAS 102, 14593 (2005)

[4] A. Zakharova et al., PLoS ONE 6, 60 (2011).

[5] A. Longtin et al., Phys. Rev. A 41, 6992 (1990); A. Longtin, Phys. Rev. A 44, 4801 (1991).

[6] K. Mergenthaler and R. Engbert, Phys. Rev. Lett. 98, 138104 (2007)

[7] J.L. Cabrera and J.G. Milton, Phys. Rev. Lett. 89, 158702 (2002)

[8] G. Deco et al., PNAS 106, 10302 (2009) ; N. Brunel and V. Hakim, Neural Comp. 11, 1621 (1999); B. Doiron et al., Phys. Rev. Lett. 93, 048101 (2004).

[9] S. Guillouzic, I. L'Heureux, and A. Longtin, Phys. Rev. E 59, 3970 (1999); T. D. Frank, Phys. Rev. E 72, 011112 (2005)

[10] R. Kuske, J. Vib. Control 16, 983 (2010).

[11] T. Ohira and J.G. Milton, Phys. Rev. E 52, 3277 (1995)

[12] L.S. Tsimring and A. Pikovsky, Phys. Rev. Lett. 87, 250602 (2001) 232, 1 (2007).

[13] B.F. Redmond, V.G. LeBlanc and A. Longtin, Physica D 166, 131 (2002).

[14] M. Gaudreault, F. Drolet, and J . Vinals. Phys. Rev. E 82, 051124 (2010)

[15] J. Hale and S. Lunel, Introduction to functional differential equations (Springer, Berlin, 1993)

[16] W. Wischert et al., Phys. Rev. E 49, 203 (1994)

[17] O. Diekmann and S. van Gils, In: Lecture Notes in Mathematics 1463, 122 (1991).

[18] M. Schanz and A. Pelster, SIAM J. Applied Dyn. Syst. 2(3), 277 (2003)

[19] P. Boxler, Probab. Theory Relat. Fields 83, 509 (1989).

[20] J. Lefebvre et al., in preparation.

[21] A. Hutt, A. Longtin, and L. Schimansky-Geier, Phys. Rev. Lett. 98, 230601 (2007); A.Hutt, Europhys. Lett. 84, 34003 (2008) 